\title{
The Indirect Determination of Stability, Heat and Momentum Fluxes in the Atmospheric Boundary Layer from Simple Scalar Variables During Dry Unstable Conditions ${ }^{1}$
}

\author{
J. E. Tillman \\ Dept. of Atmospheric Sciences, University of Washington, Seattle 98195
}

(Manuscript received 3 January 1972, in revised form 28 March 1972)

\section{ABSTRACT}

\begin{abstract}
Turbulence data from two sites have been analyzed to obtain an indirect relation between the Obukhov stability parameter, $z / L$, and temperature statistics during dry unstable conditions. The skewness of a single scalar variable, temperature, at a single height is used to obtain $z / L$, thereby demonstrating the value and importance of the no-Gaussian properties of the temperature statistics.

A relation between $\sigma_{\theta} / T_{*}$ and $z / L$ has been obtained for the stability range $-60<z / L \leqslant 0.5$. Explicit forms for heat flux and shear stress have been obtained for the unstable portion of this range in terms of $z / L, k, \sigma_{\theta}, z$, $\bar{\theta}$ and constants. Combining the above techniques provides a method of obtaining $z / L, H / \rho c_{p}$ and $u_{*}$ from temperature statistics during dry unstable conditions. Within the limitations of the observational uncertainties, the functions appear to be consistent for one or more different sites.
\end{abstract}

\section{Introduction}

Among the variables involved in the turbulent fluxes of heat, momentum and moisture in the boundary layer, temperature fluctuations probably are the most "unusual," especially under unstable conditions. Unusual is used in the sense that the temperature fluctuations are intermittent in the magnitude of the variations and generally depart significantly from a "noisy" or Gaussian process. Also, the variations have a distinct asymmetry with respect to time. Fig. 1 illustrates some of these characteristics and an experienced observer could easily separate the temperature trace from any of the wind components and could identify the direction of the time axis and the direction of hot or cold fluctuations. Given several traces during the same period at different heights or for different periods and stabilities, it would be possible to rank the instability of the various traces. Although these qualitative characteristics of the temperature variations have been observed for a number of years, very little quantitative study has been done to characterize and exploit these "unusual" characteristics. The Round Hill data from 1960 to 1961 (Cramer et al., 1961, 1962, 1966) were used to compute the first four moments or the probability distributions of $u, v, w$ and $\theta$ after subjecting the data to filtering by the removal of various running means. The computations were performed by the U. S. Army's Research Division of the Atmospheric Sciences Laboratory, Fort Huachuca, Ariz., and by the Round Hill group, but the only known publication which includes the skewness of temperature (as opposed to the skewness

${ }^{1}$ Contribution No. 255, Department of Atmospheric Sciences, University of Washington. of $d \theta / d t$ ) is Pries and Appleby (1967). The goal of the present contribution is to use the "unusual" properties of the temperature variations, as represented by the first three moments of the probability distribution of temperature, to provide the only turbulent variable required to characterize the stability and the heat and momentum fluxes under dry, unstable conditions. These techniques may prove useful when cost or complexity preclude the direct measurement of the stability or fluxes or their indirect determination via the profiles of temperature and wind speed. The direct computation of the stress is difficult since a few degrees error in the alignment of the vertical axis can cause large errors in the stress and also because the direct measurement requires the covariance of two components of a vector. Indirect determinations via profile techniques are inconvenient in that they require at least three levels of measurement of two or more variables with commensurate cost and complexity.

The basic definitions and symbols for these relations are listed below:

$$
\begin{aligned}
z / L & =-\frac{k g z H}{\rho c_{p} \bar{\theta} u_{*}^{3}} & \text { Obukhov stability parameter } \\
u_{*} & =(\tau / \rho)^{\frac{1}{2}}=\left(\overline{-u^{\prime} w^{\prime}}\right)^{\frac{1}{2}} & \text { Friction velocity } \\
H & =\rho c_{p} \overline{w^{\prime} \theta^{\prime}} & \text { Heat flux } \\
T_{*} & =-\frac{H}{\rho c_{p} u_{*}}=-\frac{\overline{w^{\prime} \theta^{\prime}}}{u_{*}} & \\
& =\frac{u_{*}^{2} \bar{\theta}}{k g L} &
\end{aligned}
$$




\section{List of Symbols}

$\begin{array}{ll}\theta & \text { potential temperature } \\ z & \text { height } \\ c_{p} & \text { specific heat at constant pressure } \\ \rho & \text { air density } \\ g & \text { gravity }\left(9.8 \mathrm{~m} \mathrm{sec}^{-2}\right) \\ k & \text { von Kármán's constant }(0.35) \\ u^{\prime} & \text { longitudinal turbulent velocity fluctuation } \\ w^{\prime} & \text { vertical turbulent velocity fluctuation } \\ \tau & \text { shear stress } \\ \sigma_{\theta} & \text { standard deviation of temperature } \\ R & \text { universal gas constant } \\ S_{T} & \text { skewness of temperature } \\ p & \text { pressure } \\ C_{1} & \text { universal constant from } \sigma_{\theta} / T_{*} \text { relation } \\ C_{3} & \text { constant from } \sigma_{\theta} / T_{*} \text { relation }\end{array}$

The prime denotes a fluctuation around a mean value while the overbar indicates the average value or mean of the quantity.

\section{Measurement program}

\section{a. M.I.T. Round Hill Field Station, South Dartmouth, Mass.}

The instrumentation and measurement program for the Round Hill site along with raw analysis have been extensively documented (Cramer et al., 1961, 1962, 1966) and the present study uses data from the $40 \mathrm{~m}$ tower, denoted by $T_{1}$.

Measurements of $u, v$ and $w$ were obtained from bivanes while temperature was measured with 0.001 inch diameter platinum resistance temperature transducers at 16 and $40 \mathrm{~m}$. Fast humidity measurements were obtained for a limited number of runs using a dual-channel microwave refractometer. Wind speed and aspirated wet and dry bulb temperature data were obtained at levels of $0.25,0.5,1.0,2.0,4.0,8.0,16.0,32.0$ and $40.0 \mathrm{~m}$ along with additional wet and dry bulb temperature data at $0.12 \mathrm{~m}$. Soil temperature profiles were obtained at depths of 1.6, 3.1, 6.2, 12.5, 25.0 and $50.0 \mathrm{~cm}$ at two horizontal locations along with soil heat flux. Solar and net radiation wère also measured.

This site could be characterized as a coastal site with moderate variations in height inland of the site. The highest terrain features within $50 \mathrm{~km}$ of the site are generally limited to heights $\leqslant 30 \mathrm{~m}$ with typical scales being on the order of $10 \mathrm{~m}$. The major local topographical feature is a ridge $\sim 20 \mathrm{~m}$ high, about $1 \mathrm{~km}$ to the west of $T_{1}$, while Buzzards Bay is $100 \mathrm{~m}$ south of the site with open ocean beyond. The data used in this study include azimuth directions between $288^{\circ}$ and $324^{\circ}$, which are the predominant directions for moderate offshore winds under fair weather conditions. These directions include homogeneous flat terrain with short grass or stubble within several hundred meters upwind followed by houses and trees beyond $300 \mathrm{~m}$. Terrain and vegetation characteristics both indicate that $z_{0}$ should vary significantly upwind of the site. Summarizing, the Round Hill site represents an example of a typical, as opposed to an ideal, site.

\section{b. Bonneville Salt Flats, near Wendover, Utah}

This site consists of crystalline salts extending many kilometers in each direction. Excluding the instrumentation vans, the largest local roughness elements (within $1 \mathrm{~km}$ ) have surface height variations on the order of $1.0 \mathrm{~cm}$ peak to valley maximum. From $1.0-2.0 \mathrm{~km}$ or more from the site there are rougher areas with heights on the order of $2 \mathrm{~cm}$ and a dike about $2 \mathrm{~m}$ high and a few meters wide. A north-south mountain ridge is approximately $40 \mathrm{~km}$ west of the site with maximum heights of about $1400 \mathrm{~m}$ and a NE-SW range with heights varying between 300 and $1000 \mathrm{~m}$ is approximately $10 \mathrm{~km}$ northwest of the site. From northeast to south the terrain is essentially flat for at least $50 \mathrm{~km}$ excluding a highway a few meters in height running east-west about $4 \mathrm{~km}$ south of the site. In terms of surface roughness, this site may be considered almost ideal.

Temperature measurements were obtained from a 10channel platinum temperature transducer system designed and constructed by the author. The basic transducer consists of a $5 \mathrm{~cm}$ long (10 $\Omega$ at 0C) $25.4 \mu$ diameter platinum resistance temperature transducer. Its characteristics are a response time limited by the time constant of the wire and a noise level of approximately $0.001 \mathrm{C} / \sqrt{\tau}$ where $\tau$ is the effective averaging time of any filtering in seconds. Long-term stability excluding errors due to wind induced strain of the platinum wires is on the order of $0.01 \mathrm{C}$ per week or better and the system has not varied by more than $0.025 \mathrm{C}$ over three years. Supporting measurements were obtained by the University of Washington's (UW) roving probe temperature and wind speed system (Paulson, 1970), and by sonic aneomometers.

\section{Observations and results}

a. Skewness of temperature and $z / L$

The basic measurements for the relation between the skewness of temperature and $z / L$ were performed by taking the skewness of the temperature after the removal of trends and low-frequency components by subtracting a running mean.

The skewness of a sampled variable $x_{i}$ is defined as

$$
S_{x}=\frac{1}{\sigma_{x}}\left[\frac{1}{n}-\sum_{i=1}^{n}\left(x_{i}-\tilde{x}\right)^{3}\right]
$$

where

$$
\bar{x}=\frac{1}{n} \sum_{i=1}^{n} x_{i}
$$




$$
\sigma_{x}^{2}=\frac{1}{n} \sum_{i=1}^{n}\left(x_{i}-\bar{x}\right)^{2}
$$

$\bar{x}$ and $\sigma^{2}$ generally being referred to as the mean and variance of the variable $x_{i}$. For a Gaussian process, $S_{x}=0$ and $S_{x}=0$ for all processes which have probability distributions that are symmetrical about a mean value. The running mean of a variable $x_{i}$ is defined as

$$
x_{i, m}=\frac{1}{m} \sum_{j=i-(m-1) / 2}^{i+(m-1) / 2} x_{j}
$$

where $m$ is an odd integer $\geqslant 3 ; x_{i, m}$ is generally denoted as an $m$ point running mean and is used in the present case for low-frequency removal by forming the new data set: $x_{k}=x_{i}-x_{i, m}$, where the new set contains the points from $1+(m-1) / 2$ to $n-(m-1) / 2$.

For the Round Hill data, the experiments were from 60-75 min long, sampled every $1.2 \mathrm{sec}$ and recorded digitally. Values of $z / L$ were computed from the heat and momentum flux measurements by removing a 6-min running mean from the raw variables. This was done to reduce the effects of non-stationary processes due to trends and very low frequency components in the basic variables. The data from this site includes the effects of site inhomogeneities and the effects of a lack of perfect leveling of the transducers. ${ }^{2}$

The Utah measurements were obtained at heights of approximately $0.55,2$ and $8 \mathrm{~m}$. Temperature data were recorded in analog form after low-pass filtering with a 3-db frequency of $6 \mathrm{~Hz}$ and subsequently digitized at $12.5 \mathrm{~Hz}$. Values of $z / L$ were computed from wind speed and temperature profiles obtained from the University of Washington roving probe system using the BusingerDyer profile relations as described by Paulson (1970). The profile estimates of $z / L$ are for runs of $\sim 40 \mathrm{~min}$.

Typical temperature traces for high instability are shown in Fig. 1 at several heights. Of particular importance are the hot spikes and the intervals during which the temperature is "quiscent" which also coincide with the coldest observed temperatures. Over periods of several minutes, the coldest temperature will recur during quiescent intervals and the minimum temperatures will generally fall within the same $0.1-0.2 \mathrm{C}$ range.

Fig. 2 illustrates the probability distribution of temperature for two Round Hill runs, of different stability, after the removal of a 72-sec running mean. Although it is only moderately unstable, Run 67D at $40 \mathrm{~m}$ clearly departs from a Gaussian process. The truncation of the cold portion of the distribution, due to the downward advection of cold-well mixed elements from greater heights, is already evident. Also evident is the long warm tail of the distribution due to the penetration of hot elements or plumes from near the surface. Both of

\footnotetext{
${ }^{2}$ The leveling effects probably are small, compared to the site effects since the leveling was accurate to within $1^{\circ}-3^{\circ}$ and the raw variables were filtered after coordinate transformations and before the fluxes were computed.
}

these processes tend to produce a positive value of skewness compared to the zero value of a Gaussian process. A similar behavior will persist over longer intervals but the temperature may vary during the quiescent periods by more than $0.1-0.2 \mathrm{C}$. During very stationary conditions the minimum temperature may be quite repeatable, but during statistically non-stationary conditions the minimum temperature may change significantly. If the probability distribution is taken during non-stationary conditions over a period of an hour without low-frequency removal by filtering, the skewness generally will be reduced and may vary from run to run even with constant instability. Also, the apparent sharp lower limit to the probability distribution may not be as evident due to the variation in the minimum temperature; i.e., the removal of trends and very low frequencies tends to reduce the scatter in the skewness $-z / L$ relation. Low-frequency filtering has been applied to the present study by removing a $72-\mathrm{sec}$ running mean from the data before computing the skewness. Running means of $72,120,360$, and $720 \mathrm{sec}$, as well as raw data without running mean removal, have been used to study the above effects. However, these will not be considered here other than to report that they qualitatively support the necessity of carefully considering the low-frequency aspects of time series, such as boundary layer "turbulent" exchange processes which are at best only approximately stationary. This is especially true if the best estimates of the instability are to be obtained for such quasi-stationary conditions.

Fig. 3a illustrates the skewness of temperature, $S_{T}$, during instabilities where $0.01 \leqslant-z / L \leqslant 3.0$ for both the Round Hill and Bonneville, Utah, sites. The Round Hill data consist of pairs of runs at 16 and $40 \mathrm{~m}$ and $z / L$ is computed from the flux measurements for each level independently. The Utah data consist of runs where temperature was simultaneously measured at one or more heights at approximately $0.6 \mathrm{~m}$ and at 2 and $8 \mathrm{~m}$. The stability parameter $z / L$ was determined by profile techniques for the $0.2-4 \mathrm{~m}$ layer.

Scatter in the $S_{T}$ vs $z / L$ relation can be caused by several factors. Among these are the lack of validity of the hypothesis that there is a unique relation, to errors in determining either parameter, to non-stationarity or, in the case of the Round Hill data, to a lack of horizontal homogeneity. The lack of horizontal homogeneity along with non-stationarity probably are the dominant Round Hill errors followed by those due to leveling effects. However, the scatter in the Round Hill data gives an indication of the validity of the relation for a typical inhomogeneous site. Errors in the Utah data can be caused by several factors. First, the data set for computing the skewness was generally shorter than that used for computing $z / L$ from the profiles. Second, there were spikes in the analog data that may not have been completely eliminated during the processing. Finally, and probably most important, are the errors in the de- 

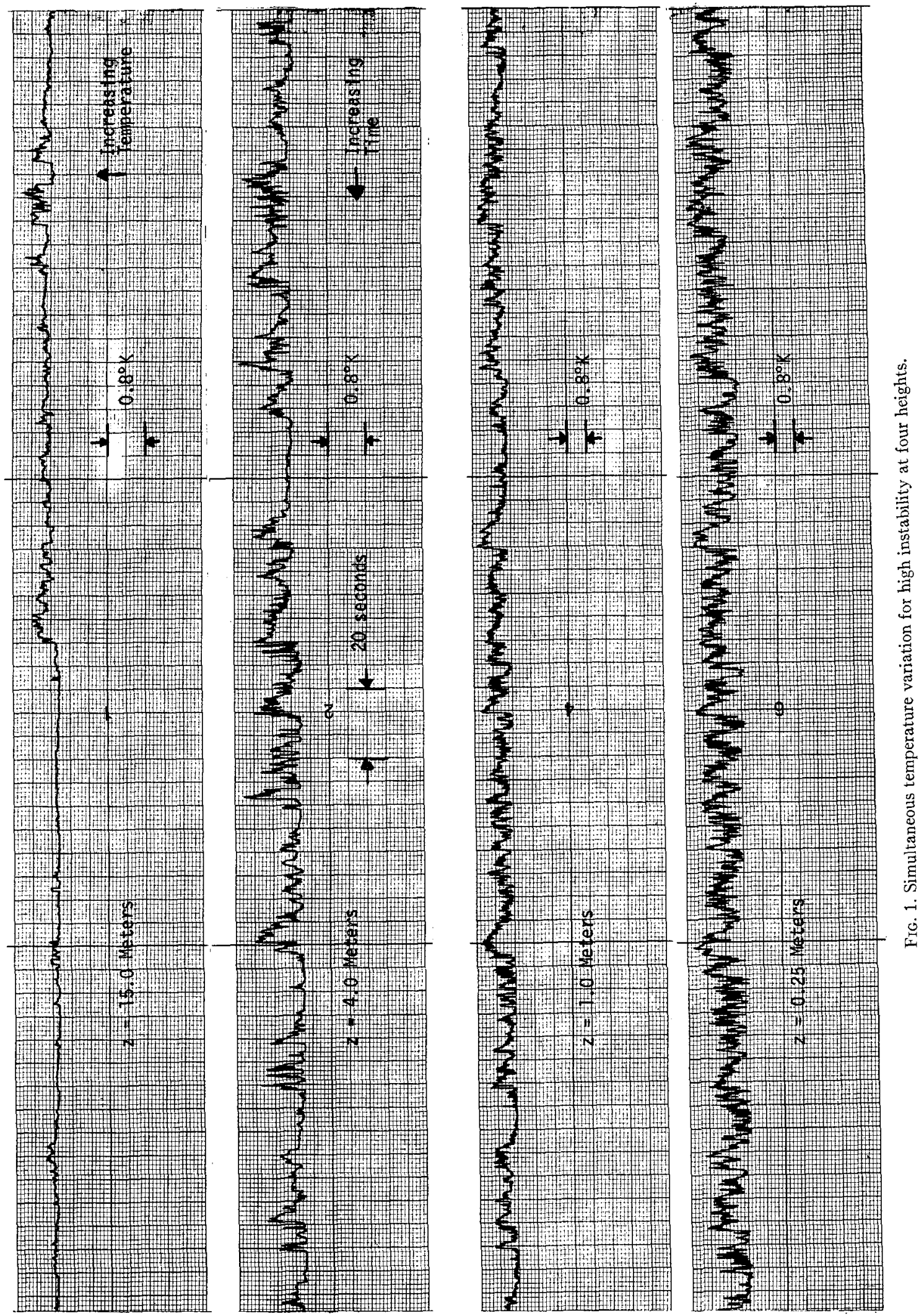


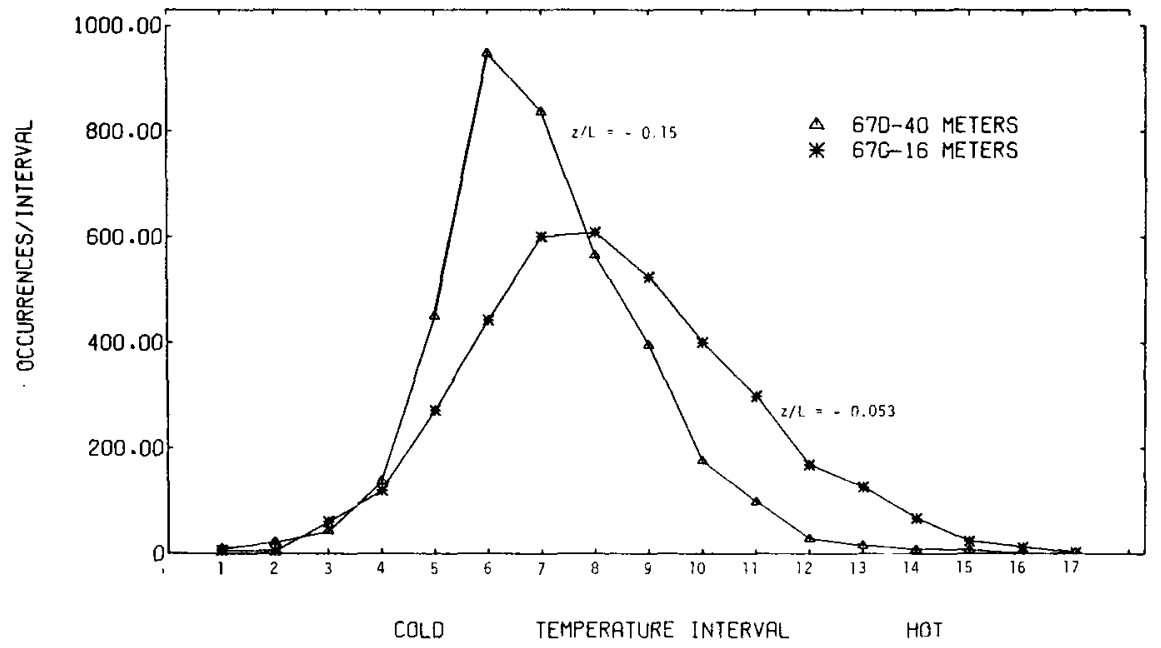

FIG. 2. Probability distribution of temperature after 72-sec running mean removal (high-pass) filtering for two Round Hill runs.

termination of $z / L$ from the profile data. A comparison between the profile determined heat flux and the heat flux measured by the sonic aneomometer-thermometer gives consistent results to within $\pm 30 \%$ or better ${ }^{3}$ excluding run 39. The profile value of heat flux for Run 39 is inconsistent with the immediately adjacent runs and the error appears to be due to a questionable gain setting on the temperature system. For this reason, the directly measured value of the heat flux was substituted for the profile value for this run.

After plotting the data in linear and semi-log form, it was determined that the semi-log form of Fig. $3 \mathrm{a}$ provided the best representation. This can be expressed as

$$
z / L=-A \exp \left(B S_{T}\right), \quad \text { for } \quad 0.01 \leqslant-z / L<3.0,
$$

where $A$ and $B$ are positive constants, obtained by taking the natural $\log$ of (5) and using a linear least square fit, assuming that errors occur both in $z / L$ and in $S_{T}$. The parameters for this data set are $A=0.0137$ and $B=4.39$ with a correlation coefficient of 0.89 using the 35 points of Fig. 3a. This function is illustrated by the solid line in Fig. $3 \mathrm{a}$ while Fig. $3 \mathrm{~b}$ compares the calculated versus measured values of $z / L$.

Although similarity theory indicates that nondimensional functions such as the skewness of temperature are exclusively determined by $z / L$, to the extent that all of the relevant variables are included, its limitations are well known. The major limitations are that it does not uniquely determine the functional form of the relation and it does not give any information as to whether the variable under consideration is a strong or weak function of $z / L$. This latter aspect is important to the extent that it is desirable to obtain $z / L$ from the simplest measurement, that can be obtained more accurately, with less cost and complexity than a direct measure-

\footnotetext{
${ }^{3}$ N. Monji, 1971, private communication.
}

ment of $z / L$ can be obtained. The relation given by (5) (or a similar one incorporating more extensive data) should be of practical value since it involves the single

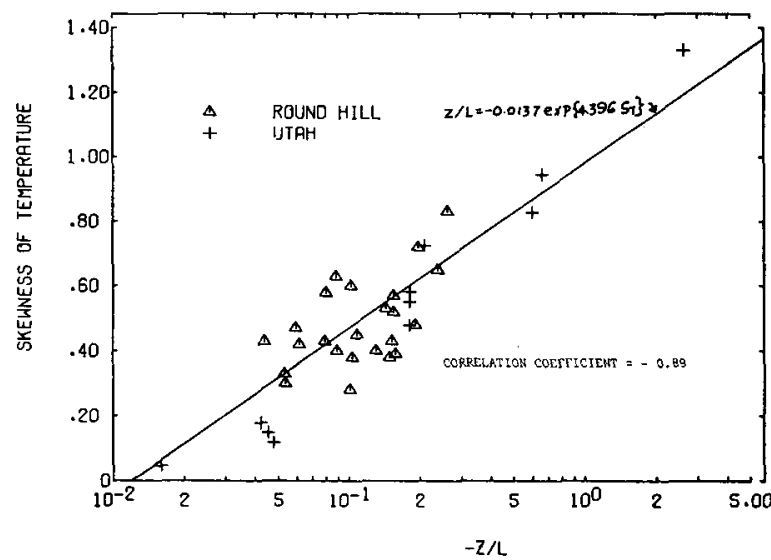

Fig. 3a. Skewness of temperature vs $-z / L$ for Round Hill and Utah unstable data.

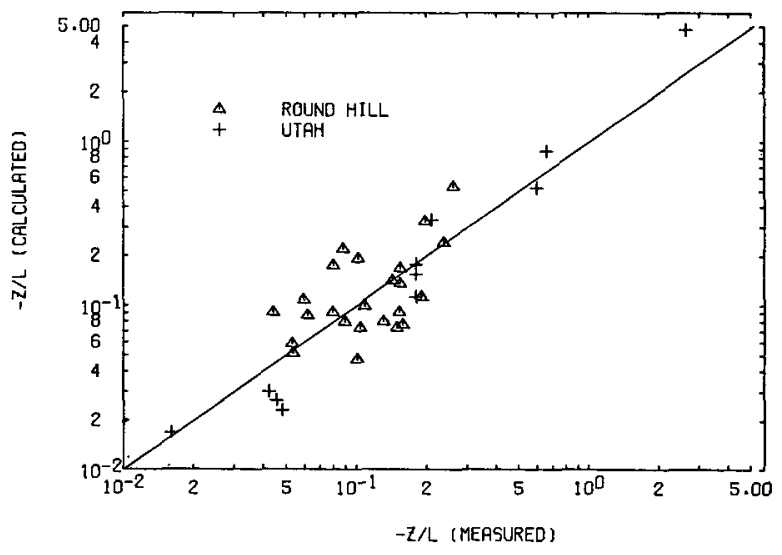

Fig. 3b. Measured vs calculated values of $-z / L$. 


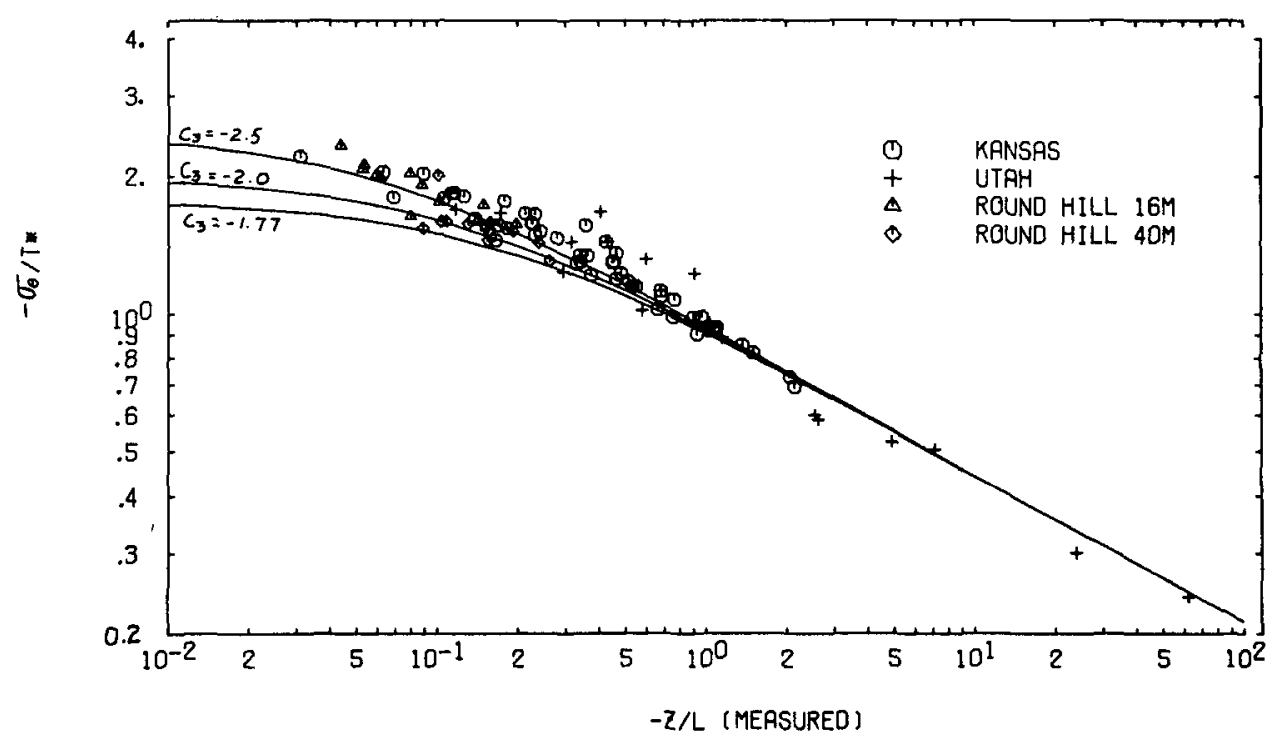

FIG. 4. $\left(-\sigma_{\theta} T_{*}\right)$ vs $(-z / L)$ for three sites, and calculated values for (8) with different values of $C_{3}$.

scalar variable temperature (which can be easily measured), is independent of calibration, and is a strong enough function of $z / L$ to be useful. With the present data, the constants of (5) can only be considered approximate and they are inadequate to distinguish between (5) and

$$
z / L=A-A \exp \left(B S_{T}\right),
$$

which might be appropriate if the skewness of temperature is zero for neutral conditions.

\section{b. Heat and momentum fluxes}

An important factor in a number of applications is the determination of the fluxes of heat and momentum. These fluxes can be obtained by the development of relations involving temperature statistics, constants and $z / L$; the substitution of (5) then reduces the problem. to the measurement of temperature statistics within the accuracy that the constants of (5) can be determined. To obtain a relation which is valid for the widest range of stability, the relation between $\sigma_{\theta} / T_{*}$ and $-z / L$ will be extended to cover the neutral and part of the stable range.

For the limiting case of free convection, similarity theory suggests a relation of the form

$$
\sigma_{\theta} / T_{*} \propto(-z / L)^{-\frac{1}{3}} \text {. }
$$

The $-\frac{1}{3}$ power law has been observed to hold for values of $-z / L \gtrsim+0.2$ and for this region of instability a formulation of the following type has been obtained ${ }^{4}$ [Perepelkina, 1962; Obukhov, 1960; Wyngaard et al.,

${ }^{4}$ Cramer and Record (1969) presented the data graphically, including von Kármán's constant in the definition of $T_{*}$, but they could not obtain a value for $C_{1}$.
1971; Monji (loc. cit)]:

$\sigma_{\theta} / T_{*}=-C_{1}(-z / L)^{-\frac{z}{3}}$ Free convection formulation $(\tau)$

Values of $C_{1}$ obtained by the above authors range from 0.90 to 1.1 . It should be noted that this relation holds well into the stability region where the shear stress has not decreased significantly from its value at the surface. Consequently, the use of the term free convection to describe the range of validity of (7) is not rigorous.

For smaller values of $-z / L$, less than $0.2,(7)$ deviates from the data and for values of instability approaching neutral, $\sigma_{\theta} / T_{*}$ appears to approach a constant. This behavior is illustrated in Fig. 4 which includes the Round Hill data of this study, additional Utah data, and the Kansas data of Wyngaard et al. ${ }^{5}$ For the complete unstable region, a formulation of the form

$$
\sigma_{\theta} / T_{*}=-C_{1}\left(C_{2}-z / L\right)^{-\frac{1}{3}}
$$

is proposed which assumes that $\sigma_{\theta} / T_{*}$ approaches a constant for neutral conditions, i.e., if

$$
C_{3}=\sigma_{\theta} / T_{*} \quad(z / L=0),
$$

then

$$
C_{2}=-\left(C_{1} / C_{3}\right)^{3} .
$$

The data of Fig. 4 indicate that the value of 0.95 for $C_{1}$ obtained by Wyngaard et al. is a good choice and Monji (loc. cit.) independently obtained 0.92 for $C_{1}$ for the Utah data. Consequently, a value of $C_{1}=0.95$ has been selected. Using this value, several values of $C_{3}$ were used to compute (8) and these are illustrated by the solid lines of Fig. 4. If $\left|\sigma_{\theta} / T_{*}\right|$ increases mono-

${ }^{5}$ To provide compatibility between Round Hill and Kansas results, a value of 0.35 was used for von Kármán's constant in the present analysis of the Round Hill data. 
tonically with decreasing instability, the data indicate that $\left|C_{3}\right| \geqslant 2.0$.

Returning to (8) and the definition of $T_{*}$, an equation for $H / \rho c_{p}$ can be obtained in the form

$$
\left(\sigma_{\theta} / C_{1}\right)\left(C_{2}-z / L\right)^{\frac{1}{3}}=H /\left(\rho c_{p} u_{*}\right) .
$$

Substituting for $u_{*}$ from the definition of $L$ gives

$$
\left(\frac{H}{\rho c_{p}}\right)^{\frac{3}{3}}=\frac{\sigma_{\theta}}{C_{1}}\left(-\frac{k g L}{\bar{\theta}}\right)^{\frac{1}{3}}\left(C_{2}-z / L\right)^{\frac{3}{3}}
$$

With the introduction of $z / z$, the following form is obtained for the heat flux:

$$
\frac{H}{\rho c_{p}}=\left[\left(\frac{\sigma_{\theta}}{C_{1}}\right)^{3}\left(\frac{k g z}{\bar{\theta}}\right) \frac{\left(C_{2}-z / L\right)}{(-z / L)}\right]^{\frac{1}{2}} .
$$

The data of Fig. 4 indicate that (11) is valid over the range $0.03 \leqslant-z / L \leqslant 60$ and may be valid for $-z / L>60$. Assuming a value of -2.5 for $C_{3}$, the resulting value of $C_{2}$ is 0.0549 and the free convection approximation to (11) should be valid for $0.05 \ll-z / L<60$. The free convection form of (11) is obtained by considering the limit for $-z / L \gg C_{2}$, giving

$$
\frac{H}{\rho C_{p}}=\left[\left(\frac{\sigma_{\theta}}{C_{1}}\right)^{3} \frac{k g z}{\bar{\theta}}\right]^{\frac{1}{2}} .
$$

The final step in the derivation of the indirect heat flux function involves the substitution of (5) for $z / L$ in (11); this results in

$$
\frac{H}{\rho c_{p}}=\left\{\left(\frac{\sigma_{\theta}}{C_{1}}\right)^{3}\left(\frac{k g z}{\bar{\theta}}\right) \frac{\left[C_{2}+A \exp \left(B S_{T}\right)\right]}{A \exp \left(B S_{T}\right)}\right\}^{\frac{3}{2}}
$$

for the forced and free convection range and in (12) for the free convection approximation.

The accuracy of (12) and (13) can be assessed from Figs. 5 and 6 which illustrate the free convection ap-

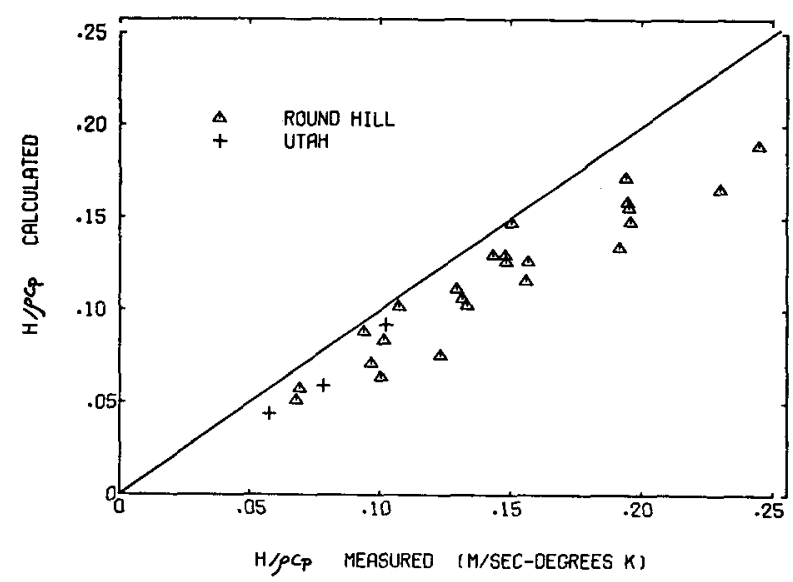

Fig. 5. Calculated vs measured heat flux using the free convection approximation for $\sigma_{\theta} / T_{*}$.

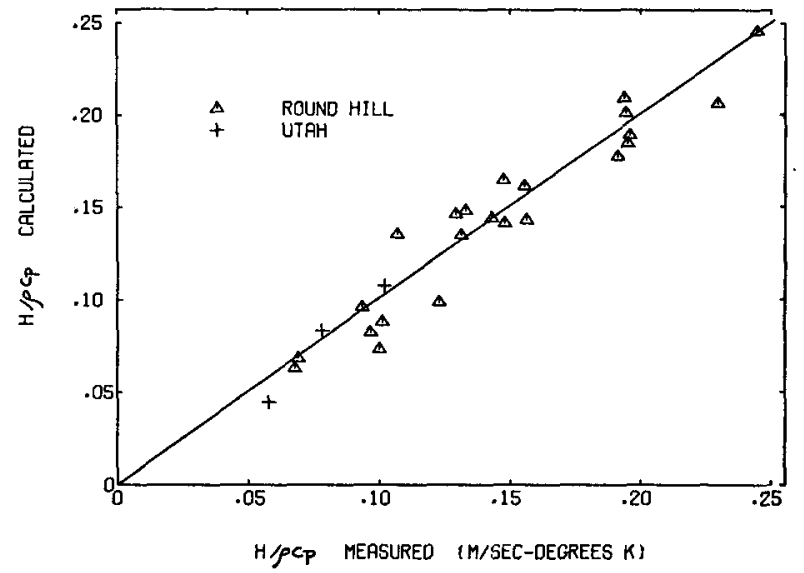

FIG. 6. Calculated vs measured heat flux using the complete unstable form for $\sigma_{\theta} / T_{*}$.

proximation and the more general unstable relation, respectively. The measured values for the Round Hill data consist of direct turbulent heat flux measurements while those for the Utah data were derived from profile data excluding one value which was obtained directly from the turbulent measurements. The calculated values for the Round Hill data were derived directly from the temperature variance and the skewness of the same transducer. For the calculated Utah data, the skewness of the temperature measurements was taken from the platinum wire transducers located at heights of $0.5,2$ and $8 \mathrm{~m}$, while the values for $\sigma_{\theta}$ were obtained from 0.005-inch diameter thermocouples located in the three-dimensional sonic aneomometer array at $4.29 \mathrm{~m}$. Consequently, skewness at $4.29 \mathrm{~m}$ was obtained by interpolation between values at other heights for each run.

Fig. 5 illustrates the limitation of the free convection approximation for these data. A perfect correlation is indicated by the straight line and the indirect calculations are consistently lower than the measured values. Fig. 6 illustrates the improvement obtained by the forced and free convection formulation for these data, which is mainly in the instability range where both mechanical and bouyant processes contribute significantly to the turbulent motions responsible for the heat flux. The formulation of (11) is physically satisfying in that for moderately unstable values of $z / L$, the heat flux is an explicit function of both the bouyant production, via $\sigma_{\theta}$, and the "forced convection" contribution, via the stability term $\left(C_{2}-z / L\right) /(-z / L)$. As the instability increases, approaching free convection, the stability dependence of (11) disappears and the heat flux is dependent on $\sigma_{\theta}, \bar{\theta}$ and the constants, and is independent of the surface shear stress $u_{*}{ }^{2}$, in agreement with similarity theory. Consequently, it should be possible to make aircraft measurements of the vertical heat flux using only the measurement of $\sigma_{\theta}$ in the free convection range where (12) is valid. For values of 
$-z / L \gg C_{2}$, the stability correction can be ignored and the validity of (5) is of no consequence in the practical determination of the heat flux.

A relation similar to (11) for the friction velocity $u_{*}$ can be obtained by substituting for $H /\left(\rho c_{p} u_{*}\right)$ in (10) from the definition of $z / L$, giving

$$
u_{*}=\left[\left(\frac{k g z}{\bar{\theta}}\right)\left(\frac{\sigma_{\theta}}{C_{1}}\right) \frac{\left(C_{2}-z / L\right)^{\frac{1}{3}}}{(-z / L)}\right]^{\frac{1}{2}}, 0.03 \leqslant-z / L<3.0,
$$

while for the range of high instability (14) reduces to

$$
u_{*} \approx\left[\left(\frac{k g z}{\bar{\theta}}\right)\left(\frac{\sigma_{\theta}}{C_{1}}\right)\right]^{\frac{1}{2}}(-z / L)^{-\frac{1}{3}}
$$

Unlike the heat flux, $u_{*}$ is, as it should be, explicitly dependent on both $\sigma_{\theta}$ and stability throughout the range of validity of (14). Substituting (5) for $z / L$ in (14) gives

$$
u_{*}=\left\{\left(\frac{k g z}{\bar{\theta}}\right)\left(\frac{\sigma_{\theta}}{C_{1}}\right) \frac{\left[C_{2}+A \exp \left(B S_{T}\right)\right]^{\frac{1}{3}}}{A \exp \left(B S_{T}\right)}\right\}^{\frac{1}{2}},
$$

which results in a functional form for $u_{*}$ involving the same parameters as (13). Figs. 7 and 8 compare the indirect $u_{*}$ vs the measured $u_{*}$ using Eq. (16) and its counterpart for the free convection approximation which is obtained by setting $C_{2}=0$ in (16): there is little difference between the two resulting expressions for the present data. This is expected since the difference between the partial and the complete unstable formulation is the difference between the terms $(-z / L)^{1 / 6}$ and $\left(C_{2}-z / L\right)^{1 / 6}$. The sixth root minimizes the difference over the modest range of instabilities presented.

Returning to (14) one can obtain

$$
\frac{u_{*}^{2}(-z / L)}{\sigma_{\theta}}=\frac{k g z}{\bar{\theta} C_{1}}\left(C_{2}-z / L\right)^{\frac{z}{3}} .
$$

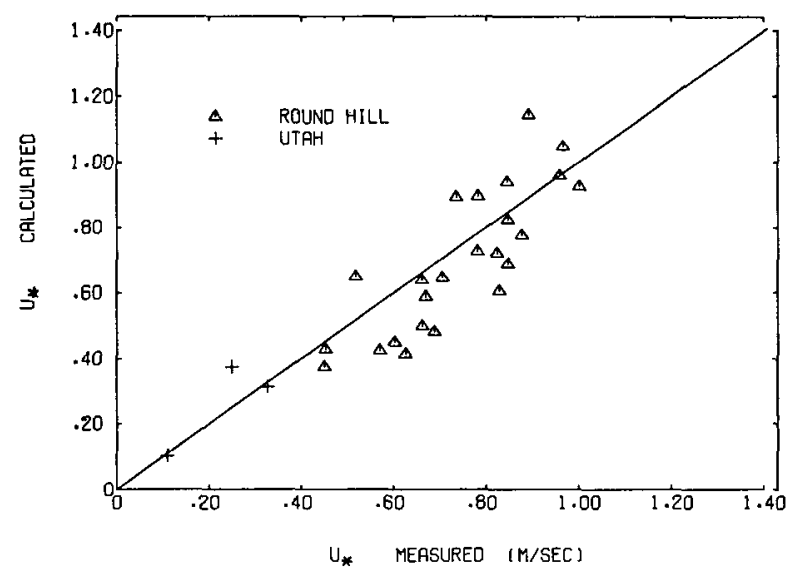

Fig. 7. Calculated vs measured $u_{*}$ using the free convection form for $\sigma_{\theta} / T_{*}$.

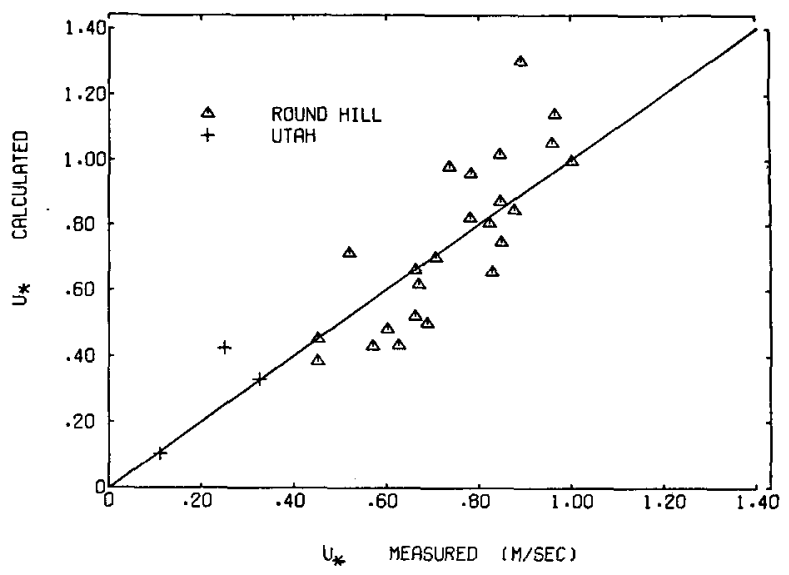

Fig. 8. Calculated vs measured $u_{*}$ using the complete unstable form for $\sigma_{5} / T_{*}$.

As $-z / L \rightarrow 0$, combining (17) and (9b) gives

$$
\frac{u_{*}^{2}(-z / L)}{\sigma_{\theta}} \approx \frac{k g z}{\bar{\theta} C_{1}}\left(C_{2}\right)^{\frac{1}{3}}=-\frac{k g z}{\bar{\theta} C_{3}} \text {. }
$$

Rewriting (18) as

$$
u_{*} \approx\left[\frac{\sigma_{\theta}}{(-z / L)}\left(-\frac{k g z}{C_{3} \bar{\theta}}\right)\right]^{\frac{1}{2}}=\left[L \sigma_{\theta} \frac{k g}{C_{3} \bar{\theta}}\right]^{\frac{1}{2}}
$$

implies that $z \sigma_{\theta} / \bar{\theta}(-z / L)$ or $L \sigma_{\theta} / \bar{\theta}$ remain finite as $-z / L \rightarrow 0$ in such a manner that $u_{*}$ is determined for neutral conditions, excluding the case where the mean wind speed is simultaneously zero; i.e., for $-z / L \ll C_{2}$ $\sigma_{\theta}$ remains constant with decreasing $z$.

For the near-neutral case, one can obtain from (9b) and (11) the relation

$$
\frac{H}{\rho c_{p}} \approx\left[\left(\frac{\sigma_{\theta}}{C_{3}}\right)^{3}\left(\frac{k g L}{\bar{\theta}}\right)\right]^{\frac{1}{2}} .
$$

Taking the ratio of (20) to (19) or from the limit of (10) for the neutral case, one has

$$
-\frac{H}{\rho C_{p} u_{*}} \equiv T^{*}=\frac{\sigma_{\theta}}{C_{3}}
$$

where, in terms of fluctuation quantities,

$$
-C_{3}=\frac{\rho c_{p} u_{*} \sigma_{\theta}}{H}=\frac{\left(-\overline{u^{\prime} w^{\prime}}\right)^{\frac{1}{2}} \sigma_{\theta}}{\overline{w^{\prime} \theta^{\prime}}} .
$$

Given the correct value of $C_{3}, \sigma_{\theta}$ and any relation [such as (5), profile relations, or other flux relations] which defines a unique separable relation between $\overline{u^{\prime} r v^{\prime}}$ and $\overline{w^{\prime} \theta^{\prime}}$, in principle one can obtain both the heat and momentum fluxes. Due to the importance of $C_{3}$, its uniqueness and numerical value should be determined.

It is difficult to determine $C_{3}$ as neutral conditions are approached in that both $\sigma_{\theta}$ and the heat flux 
approach zero and the ratio becomes increasingly harder to obtain accurately. Another implicit problem is the assumption that conditions are both stationary and homogeneous during the measurement interval. Nearneutral conditions can be obtained by taking measurements closer to the ground but this places more stringent requirements on the spatial response of the transducers and on the surface uniformity. Also, as the surface is approached, $z$ approaches the surface roughness $z_{0}$ and no complete atmospheric turbulence results are known for homogeneous sites where $z \approx z_{0}$. If near-neutral conditions are obtained by selecting runs with low heat flux due to overcast conditions (where the net radiation may vary with overcast) or to the diurnal variation of the heat flux, the data must be carefully examined to rule out the possibility that non-stationary conditions make the results inconclusive. If significant non-stationarity does exist, the present formulations of the turbulent processes are incomplete and, for example, they must include time varying values of the variables.

If additional measurements during stable conditions are considered it may be possible to obtain $C_{3}$ in a manner which does not involve the changing ratio of two variables as both variables approach zero. This may be accomplished by considering the data of Fig. 9 [from the Kansas City experiment of Wyngaard et al. (1971)] where $(-z / L) \sigma_{\theta}{ }^{2} / T_{*}{ }^{2}$ is plotted vs $z / L$. The ordinate is related to the square of the function of Fig. 4 multiplied by $-z / L$. The important point is that the data of Fig. 9 appear to vary linearly through neutral stability with a constant slope for the stable region $0.0 \leqslant z / L<0.5$. If the slope is constant through neutral, i.e., $C_{3}$ is identical on either side of neutral, then it can be determined more precisely from the stable data. It can then be shown that

$$
\left|C_{3}\right|=\left|\frac{\partial\left[(-z / L) \sigma_{\theta}{ }^{2} / T_{*}{ }^{2}\right]}{\partial(-z / L)}\right|^{\frac{1}{2}}
$$

for any region where $C_{3}$ is constant. Visually fitting a straight line to the data of Fig. 9 (biasing the fit to the stable data which also has smaller scatter) implies that $C_{3} \approx-1.77$, which is lower than the value estimated from Fig. 4. The average value of $\sigma_{\theta} / T_{*}$ for the data of Fig. 9, for the stable range $0.0 \leqslant z / L \leqslant 0.5$, gives $C_{3}=-1.80$.

The apparent discrepancy between the value of $C_{3}$ obtained from the unstable data and that from the data including the stable region as given in Fig. 9, could be due to 1) an actual difference, 2) a lack of homogeneity, 3) non-stationarity conditions, and 4) measurement errors. If inhomogeneous conditions were responsible for the discrepancy, the Round Hill data should be significantly different from the Kansas data since the Round Hill site is far less homogeneous than the Kansas site. It does not seem plausible to attribute the difference to measurement errors since the data are similar while the instruments, techniques, site and other factors differ markedly between the experiments. A lack of stationarity may be responsible for the difference in $C_{3}$ as determined by the stable, as opposed to the unstable, data. For the stable conditions, the depth of the mixed layer is smaller than for unstable conditions and adjustments to stationary conditions probably take place more rapidly as long as the stability does not become so large that breakdown occurs. Since the stability is a response to the diurnal cycle of net radiation, the large variations in net radiation during the daytime as opposed to the slower and smaller nighttime variations would also suggest that stationary conditions would be easier to obtain under stable nighttime conditions. If this difference is real and is due to non-stationary conditions, the apparent variation in $C_{3}$ (which is no longer a constant for non-stationary conditions) may be useful in evaluating the effect of non-stationarity on similarity estimates of the heat flux and shear stress by comparing

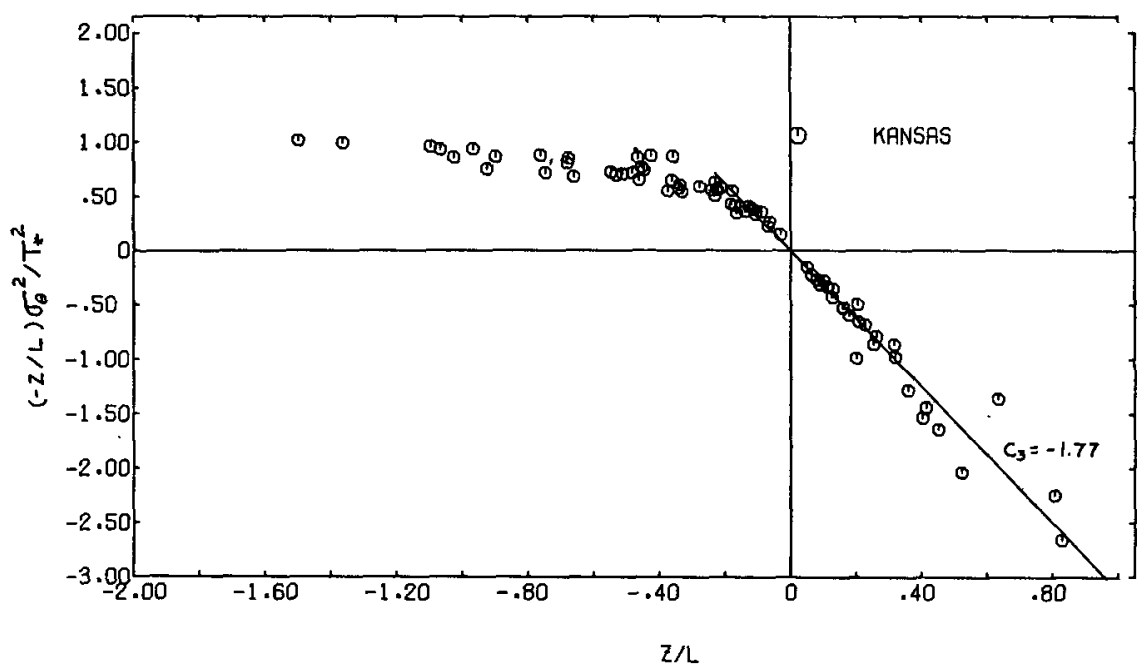

FIG. 9. Measured $(-z / L) \sigma_{\theta}{ }^{2} / T_{*}{ }^{2}$ vs measured $z / L$ for unstable and stable data. 
measured values of heat flux and shear stress with those obtained from (11) and (14) using $C_{3}=-1.80$. Finally, the difference may be real and attributable to factors other than those stated. Curves for different values of $C_{3}$ are shown in Fig. 4.

The data of Fig. 9 suggest that for positive (stable) values of $z / L \leqslant 0.5,(20)$ is valid if a negative sign is used in front of the brackets. Consequently, the indirect technique for obtaining heat flux from temperature statistics and $z / L$ covers the range of stability $-60 \leqslant z / L \leqslant 0.5$, while temperature statistics alone appear adequate for the stability range $-60 \leqslant z / L \leqslant-0.02$.

As the value of von Kármán's constant is presently in doubt (Businger et al., 1971), the heat flux probably can be determined more accurately from the raw data with new constants replacing von Kármán's constant and the constants $C_{1}$ and $C_{3}$. This should also be true for the stress since the same constants appear in this relation. To the extent that moisture fluxes are small, these indirect techniques for the determination of $z / L$, heat and momentum fluxes during unstable conditions may provide adequate accuracy in many applications without the necessity of direct flux measurements or their indirect determination by profile techniques. The density may be replaced by a mean pressure term and a mean temperature in any of the above equations via the perfect gas law. With the present measurements, the unstable region is best described by $C_{3}=-2.5$ while the near-neutral portion of the stable region suggests $C_{3}=-1.77$. An assessment of the differences as to the importance of non-stationarity, etc., will require other experimental data.

The skewness computation does not require a measurement of either the absolute value of the temperature or the magnitude of the temperature fluctuations since both the mean value and any linear multiplier of the temperature measurement cancel in the determination of the skewness. The only requirement is that the measurement be essentially linear over the range of perturbations encountered - an easily satisfied requirement. In this sense, this technique is a member of a class of "gain" or "scale factor" independent functions which also are independent of the magnitude of the variable itself. Another dimensionless example is the correlation coefficient. Although the $z / L$ vs $S_{T}$ relation is tentative, it illustrates that it is reasonable to search for similar gain-independent functions for nondimensional parameters which place a modest burden on the measurement process.

One application of the stability relation would be that where the temperature fluctuations are the primary independent variable of the process being studied, such as in acoustical or optical propagation. In such applications, the non-Gaussian aspects of the temperature, as given by the skewness and higher moments, often are important to the understanding of the basic process. For these cases, the temperature measurement can characterize the stability without recourse to other measurements. If more than one temperature measurement is made to study the temporal-spatial properties of the temperature field, then independent stability estimates can be obtained from each of the transducers either for cross-checking or to estimate the magnitude of effects due to inhomogeneities.

Acknoweldments. The author would like to sincerely thank Prof. J. A. Businger for his invitations to join the University of Washington's Utah field program and to continue the analysis of this problem at the University of Washington, his suggestion to incorporate a $\sigma_{\theta} / T_{*}$ relation, and his discussions during the analysis. He would like to express his appreciation to Prof. C. B. Leovy for his encouragement and discussions, to Prof. Badgley for his incisive comments, and would like to thank Dr. O. Coté for supplying the tabulated data from the Kansas experiment collected by the Boundary Layer Branch of the Air Force Cambridge Research Laboratories. Finally he would like to acknowledge the help of Mr. Eric Leavitt in the programming and processing of the data and to all members of the Energy Transfer Group involved in the field program.

This work was partially supported by the National Science Foundation under Grant 6A31317X and the National Aeronautics and Space Administration under Contract NASI 9694.

\section{REFERENCES}

Businger, J. A., et al., 1971: Flux-profile relationships in the atmospheric surface layer. J. Atmos. Sci., 28, 181-189.

Cramer, H. E., and Record, F. A., 1969: Properties of turbulent energy spectra and cospectra in the atmospheric surface layer. Final Rept., Grant DA-AMC-36-039-64-G1, Dept. of Meteorology, M.I.T.

_- - - and J. E. Tillman, 1962: Studies of the spectra of the vertical fluxes of momentum, heat and moisture in the atmospheric boundary layer. Final Rept., Contract DA-36-039SC-80209, Dept. of Meteorology, M.I.T.

__ - - - and _- 1966: Round Hill turbulence measurements. Tech. Rept. ECOM-65-G10, Grant DA-AMC-28-043-65G10, 5 volumes, Dept. of Meteorology, M.I.T.

- - _ - — and H. C. Vaughan, 1961: Studies of the spectra of the vertical fluxes of momentum, heat and moisture in the atmospheric boundary layer. Annual Rept., Contract DA-36039-SC-80209, Dept. of Meteorology, M.I.T.

Obukhov, A. M., 1960: The structure of the temperature and velocity fields in free convection. Akad. Nauk SSSR, Ser. Geofiz., No. 9.

Paulson, C. A., 1970: The mathematical representation of wind speed and temperature profiles in the unstable atmospheric surface layer. J. Appl. Meteor., 6, 857-861.

Perepelkina, A. V., 1962: Characteristics of turbulence of the near ground layer under free convection conditions. Bull. Acad. Sci. USSR, Geophys. Ser., No. 2, 271-274.

Pries, T. H., and J. F. Appleby, 1967: The distribution of eddy velocities and temperature fluctuations in the first 100 meters. Tech. Rept. ECOM-6027, U. S. Army Electronics Command, Atmospheric Sciences Laboratory, Fort Huachuca, Ariz.

Wyngaard, J. C., O. R. Coté and Y. Izumi, 1971: Local free convection, similarity and the budgets of shear stress and heat flux. J. Almos. Sci., 7, 1171-1182. 